\title{
Simultaneous appearance of leukemoid reaction and phlegmasia cerulea dolens
}

\section{T Kovačević* ${ }^{*}$ P Kovačević $\dagger$, F Pecoraro ${ }^{\ddagger}$ and Z Rančić ${ }^{\dagger \ddagger}$}

*Centre for Anesthesia and Reanimation, Clinical Centre Nis; ${ }^{\dagger}$ Faculty of Medicine University Nis, Nis, Serbia;

${ }^{\ddagger}$ Clinic for Cardiovascular Surgery, University Hospital Zurich, Zurich, Switzerland

\begin{abstract}
A leukemoid reaction is an extreme form of reactive leukocytosis defined as granulocytic leukocytosis above $50 \times 10^{9} / \mathrm{L}$ produced by normal bone marrow, mostly in response to systemic infection or cancer. The mechanism as to how the haematopoetic system is altered to elevate production of myeloid cells is not known.

A 69-year-old man presented with phlegmasia cerulea dolens caused by massive iliofemoral thrombosis. His workout at admission revealed absolute white blood cell count of $73.4 \times 10^{9} / \mathrm{L}$, with neutrophil granulocyte of $68.5 \times 10^{9} / \mathrm{L}$. The new increase in white blood cell count happened at day 5 after admission, when the haematoma of the anteromedial thigh was evacuated in general anaesthesia. There was a gradual decrease in counts until they reached the normal range. Deteriorated general condition with signs of systemic inflammatory response syndrome improved with supportive therapy, and the patient was discharged from hospital after 30 days. During hospitalization we did not identify any infectious focus, or any malignancy. We could not exclude other occult chronic conditions (malignancy) but the patient did not develop any other condition during 4.5 years of follow-up.
\end{abstract}

Keywords: phlegmasia cerulea dolens; leukemoid reaction

\section{Introduction}

Phlegmasia cerulea dolens (PCD), first described four centuries ago by Fabricius Hildanus, is a rare clinical manifestation of deep iliacofemoral vein thrombosis. Thrombosis involves superficial and deep veins, as well as collateral veins resulting in venous congestion. This condition leads to massive fluid sequestration and severe painful oedema. The arterial system could be compromised, and the situation can deteriorate into manifest venous gangrene. ${ }^{1}$

Leukemoid reaction (LR) is a benign haematological disorder defined as granulocytic leukocytosis above $50 \times 10^{9} / \mathrm{L}$ with hyperactivity of maturated bone marrow mostly induced with systemic infection or cancer. The first diagnostic task is to

Correspondence: Z Rančić, Raemistrasse 100, 8032 Zurich, Switzerland.

Email: zoran.rancic@usz.ch

Accepted 11 July 2011 exclude chronic granulocytic leukaemia and chronic neutrophilic leukaemia or other myeloproliferative neoplasm. The LR can be seen in Down's syndrome, ${ }^{2}$ and was described in low-birth-weight infants. ${ }^{3}$ The main causes for LR are: severe infection (colitis due to clostridium difficile, disseminated tuberculosis and shigellosis or salmonella infection, pyogenic infections), burns, tissue necrosis ${ }^{4-7}$ and malignancies (carcinoma of the lung, thyroid, oropharinx, gastrointestinal or genitourinary tract, in patients with Hodgkin's disease, melanoma, soft tissue sarcoma, differentiated liposarcoma or hepatic angiosarcoma). ${ }^{4,7-9}$ Severe haemorrhage as retroperitoneal haemorrhage also can be associated with $\mathrm{LR}_{r}^{4,10,11}$ intoxication alcoholic hepatitis or lithium medication. ${ }^{9,12}$

\section{Case report}

A 69-year-old retired lorry driver (with a body mass index [BMI] of $35 \mathrm{~kg} / \mathrm{m}^{2}$ ) was admitted to the emergency department with massive left leg swelling, 
cyanosis of the foot involving toes and plantar aspect, non-palpable foot pulses (normal foot pulses in other limb), with numbness and sensory loss, as well as 'muscle weakness' with loss of toe movements. The patient first experienced discomfort in the left leg two days prior, with gradual progression of pain, coldness and swelling below and thereafter above - the knee, which prohibited further movements. He was hospitalized five years prior because of posterior tibial and peroneal deep venous thrombosis (DVT) treated with heparin followed by oral anticoagulants for three months and acetylsalicylic acid $100 \mathrm{mg}$ thereafter. From the patient history it was realized that the primary episode of DVT was provoked by eight hours of continuous highway truck driving. He had no other underlying diseases.

On admission the patient was confused but conscious and pale; the vital signs taken from him included heart rate $92 \mathrm{bpm}$, blood pressure 110/ $60 \mathrm{mmHg}$ and respiratory rate 25 breaths per minute. There were no other alterations in physical findings. Initial Doppler ultrasonography (US) revealed a left external iliac-femoropopliteal DVT. Computed tomography (CT) angiography excluded pulmonary embolism, retroperitoneal bleeding, May-Thurner syndrome, other cause of iliac vein compression, but confirmed extensive DVT with partially occluded left common iliac vein, and thrombus-free inferior vena cava. Routine haematology and biochemistry examinations showed total white blood cell (WBC) of $73.4 \times 10^{9} / \mathrm{L}$ with neutrophil granulocyte (NG) count of $68.5 \times 10^{9} / \mathrm{L}$, red blood cell count of $5.15 \times 10^{12} / \mathrm{L}$ and platelets of $560 \times 10^{9} / \mathrm{L}$, urea $20.2 \mathrm{mmol} / \mathrm{L}$ and creatinine $159 \mathrm{mmol} / \mathrm{L}$. The patient was referred to the haematologist: peripheral blood smear showed increased mature NG count with 'shift to the left'. Bone marrow aspiration from the right posterior iliac crest showed higher cellular marrow with intact maturity and morphology of all elements; no blasts or dysplastic features were identified.

We initiated anticoagulation treatment with intravenous heparin administration: bolus of $100 \mathrm{IU} / \mathrm{kg}$, followed by a continuous infusion of $15 \mathrm{IU} / \mathrm{kg} /$ hour. Therapeutic anticoagulation was assessed with monitoring activated partial thromboplastin time with a goal range above 2.5 times than the laboratory reference range (35-50 seconds). The patient tolerated elevation of the leg. Every four hours, his lower leg and thigh were assessed to detect compartment syndrome. After 24 hours there was no progression of swelling, cyanosis appeared to be less evident and capillary refill was shortened. The patient refused prophylactic placement of the inferior vena cava filter. There was a slight decrease in the WBC and NG counts. As the excess high leukocyte number was postulated to be LR, the use of antibiotics was restricted.

On the fifth day after admission, haematoma of the anteromedial aspect of the thigh was detected. We could not exactly specify the cause of the thigh haematoma. Haematoma was extended above and along the great saphenous vein. We excluded trauma, and anticoagulation was in therapeutic range. We presumed that massive leg swelling resulted in splitting the skin and the subcutaneous fat from the underlying fascia, with bleeding from the capillaries. A large haematoma of $10 \times 8 \mathrm{~cm}$ caused new onset of uncontrolled pain, loss of Doppler signals over the pedal arteries and inability to move the toes. We decided to decompress and evacuate haematoma; the wound was left open and packed with povidone-iodine solution gauze. According to in-hospital clinical regulation, we used second-generation cephalosporin cefuroxime for primary prevention of wound infection. Our policy was to administer antibiotics 30-60 minutes before the skin incision. In total, cefuroxime was administered for three days, and antibiotics were stopped after negative smear.

After haematoma evacuation, the patient's general condition deteriorated, and he presented with signs of systemic inflammatory response syndrome (SIRS): tachypnoea, renal impairment and 5-7 mushy water diarrhoea. We performed cultures of the stool to identify Clostridium difficile, and Shigella spp. Both cultures were negative. Symptomatic and supportive therapy led to patient recovery. The WBC and NG counts decreased in number, so that at the 29th day after admission the WBC and NG count were $9.2 \times 10^{9}$ and $4.6 \times 10^{9}$, respectively (Table 1 ). The highly sensitive C-reactive protein (hs-CRP) value decreased from $182 \mathrm{mg} / \mathrm{L}$ at time of haematoma evacuation to $13 \mathrm{mg} / \mathrm{L}$ at discharge (Figure 1). Doppler ultrasound examination was performed once a week to demonstrate re-canalization of the deep veins. At discharge the popliteal vein and deep femoral vein were completely re-canalized, while the superficial femoral (SFV) and external iliac vein (EIV) were partially re-canalized. Doppler ultrasound at 12-month control demonstrated near total re-canalization of SFV, and partial re-canalization of EIV. The patient was instructed to wear compression stockings in order to reduce postthrombotic risk and swelling of the leg. Peroneal nerve palsy was present, but capillary refill was 
Table 1 WBC, neutrophil counts and hs-CRP level during hospitalization

\begin{tabular}{|c|c|c|c|c|c|c|c|c|c|}
\hline Day & 1 & 4 & 7 & 10 & 14 & 17 & 20 & 24 & 29 \\
\hline $\mathrm{WBC} \times 10^{9} / \mathrm{L}$ & 73.4 & 68.4 & 78.5 & 60.6 & 52.5 & 37.5 & 24.2 & 17.3 & 9.2 \\
\hline Neutrophil $\times 10^{9} / \mathrm{L}$ & 68.5 & 54.3 & 65.1 & 54.5 & 48.1 & 34.2 & 21.7 & 9.8 & 4.6 \\
\hline $\mathrm{hsCRP}_{\mathrm{C}} \mathrm{g} / \mathrm{L}$ & 182 & 170 & 190 & 165 & 120 & 100 & 75 & 40 & 13 \\
\hline
\end{tabular}

normal. The patient was scheduled for 12 months of oral anticoagulation.

We saw the patient in the outpatient clinic after 4.5 years in a good general condition, with normal peripheral blood smear, with no malignancy or any infectious diseases developed in the meantime.

\section{Discussion}

LR is defined as marked granulocytic leukocytosis above $50 \times 10^{9}$ per litre produced by normal bone marrow in response to systemic infection and cancer. There is also LR drug-induced with granulocyte colony stimulating factor, corticosteroids, tetracycline or streptokinase. Also, the diabetic ketoacidosis, alcoholic hepatitis, ethylene glycol intoxication or enteric necrosis can lead to LR. Nevertheless, the major cause of LR is malignancy. By definition, LR is diagnosed after the exclusion of malignant haematological disorder, chronic myelogenous leukaemia (CML), chronic neutrophilic leukaemia $(\mathrm{CNL})$ and acute leukaemia. Hypercellular bone marrow with intact maturation and normal morphology of all elements is characteristic of LR. In CML, bone marrow shows basophilia, eosinophilia, monocytosis, slight increase in blasts and reticulin fibrosis; in CNL, bone marrow shows similar morphology with LR, packed bone

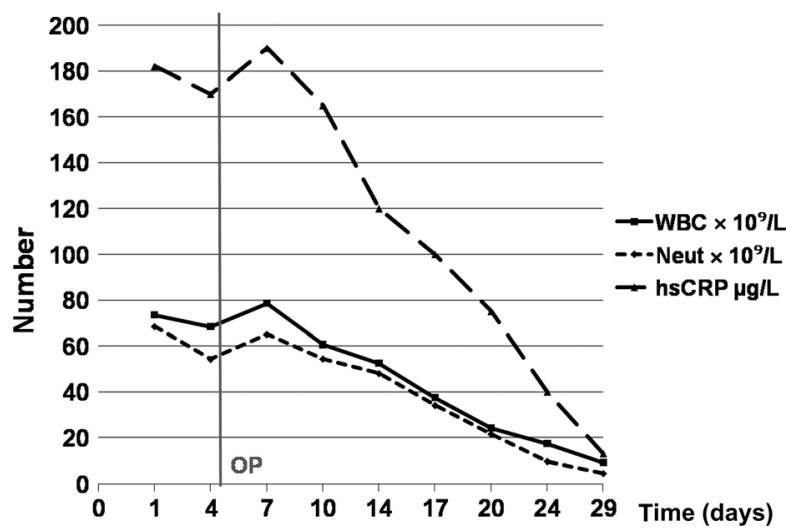

Figure 1 White blood cell (WBC), granulocyte (neutrophil) counts and hs-C-reactive protein (hs-CRP) level in patient during hospitalization. Grey vertical line represents day 5 after admission, when thigh haematoma was evacuated. Neut, neutrophil marrow, and slight increase in reticulin [Sakka]. Also, secondary involvement of bone marrow is seen in $5-15 \%$ of patients with Hodgkin's disease and $30-50 \%$ of patients with non-Hodgkin's lymphoma. Finally, bone marrow can be affected by bone metastasis of solid tumours: breast, lungs, prostate, kidney, gut and thyroid. CT and further follow-up examinations did not detect any obvious malignant disease. In our patient, bone marrow was typical for LR, and during the follow-up period either CML or CNL were presented. No other primary or metastatic malignancies were identified in the bone marrow. Diagnosis of LR was at the beginning challenging due to elevated platelet counts. Also, the erythrocyte count was just below the upper limit. Additional marked neutrophilic leukocytosis raised suspicion of myeloproliferative neoplasm. At day 2 after admission, the platelet count was normal, and careful diagnostic work-up excluded myeloproliferative neoplasm.

The aetiology of the patient's extensive iliofemoral and popliteal DVT is not clear. The first episode of DVT might be provoked by relative immobilization (continuous driving) in the obese lorry driver (BMI $35 \mathrm{~kg} / \mathrm{m}^{2}$ ). The second episode occurred without any obvious trigger. As it was the second episode of DVT with potentially devastating presentation, before starting the treatment we took blood for thrombophilia work-up. Tests were negative.

The appearance of LR after cardiovascular operation was described, but it was acute onset of myelomonocytic leukaemia. Pathology ways responsible for such response are unknown, but the authors accentuated an altered activation of inflammatory response due to the combination of surgical trauma, use of cardiopulmonary bypass and ischaemia-reperfusion syndrome. Marked amplification of this process can result in SIRS resulting in the complex and poorly understood response of the bone marrow to stress.

There are not a lot of reports on the association of vein thrombosis with increase of isolated blood cells (platelets, erythrocyte or leukocytes). Berk et al. reported a patient with massive portal, splenic and mesenteric vein thrombosis who 
presented essential thrombocytemia after splenectomy. ${ }^{15}$ The association of DVT and leukocytosis is lacking. Japanese authors described the association of LR and portal and splenic vein thrombosis as a complication of gastric ulceration. ${ }^{14}$ There are more reports of hepatic vein thrombosis that resulted in Budd-Chiari syndrome secondary to polycythaemia rubra vera (increase in total erythrocyte volume, thrombocytosis and leukocytosis). ${ }^{15,16}$

Our patient with extensive DVT presented as PCD had, at admission, extreme leukocytosis. An extended literature search did not, to the best of our knowledge, describe such simultaneous appearance of DVT and LR. We could not state which of both, if any, occurred first. We can not exclude the possibility that DVT and LR are incidentally presented at the same time, without any causal association.

On the one hand, LR could precede DVT, and after several days through some unknown inflammatory paths result in DVT.

On the other hand, LR might be seen per se as a manifestation of extensive DVT. DVT might through some mediator(s) affect bone marrow response to LR. Which mediator(s) made this cross-talk between inflammation and thrombosis possible is beyond this report. hs-CRP could play a role in this interaction, especially as its level corresponded to the leukocyte number. Of interest is that after haematoma evacuation the second increase of leukocytes occurred simultaneously with the increase of hs-CRP. One of the explanations for cross-talks between LR (leukocytosis) and DVT could be inflammation and hs-CRP as the inflammation marker. Haematoma formation and surgical trauma (for haematoma evacuation) are the inflammation triggers for increased number of leukocytes and hs-CRP production. Clinical course and leukocyte elevation simultaneously with new haematoma onset, decrease in number with time, and resolution of DVT indicate the potential association between extensive DVT and LR.

To determine the optimal duration of anticoagulation was not easy. Bearing in mind the first episode of DVT (provoked by relative immobilization) the duration of anticoagulation after second DVT should be six months. But the second episode of DVT occurred without an obvious trigger, so that we might consider indefinite duration of coagulation. In this patient, we tried to individualize treatment strategy. We took into account the facts which were in favour of indefinite anticoagulation: incomplete re-canalization of deep veins at follow-up venous ultrasound examination, PCD as a clinical presentation of DVT, and lack of an obvious trigger for recurrent DVT. Furthermore, we were aware that thrombophilia tests and hypercoagulability work-up were negative, and the patient refused anticoagulation after 12 months. Nowadays, there are other 'hybrid approaches' for optimizing the duration of anticoagulation in patients with DVT. ${ }^{17}$

During hospitalization we did not find any infectious focus, or any malignancy. We could not exclude other occult chronic conditions (malignancy) but the patient did not develop any other conditions during 4.5 years of follow-up.

\section{References}

1 Cooper RM, Hayat SA. Phlegmasia cerulea dolens, a rare complication of deep vein thrombosis. Emerg Med J 2008;25:334

2 Xu G, Nagano M, Kanezaki R, et al. Frequent mutations in the GATA-1 gene in the transient myeloproliferative disorder of Down syndrome. Blood 2003;102: 2960-8

3 Hsiao R, Omar SA. Outcome of extremely low birth weight infants with leukemoid reaction. Pediatrics 2005;116:e43-51

4 Sakka V, Tsiodras S, Giamarellos-Bourboulis EJ, Giamarellou $\mathrm{H}$. An update on the etiology and diagnostic evaluation of a leukemoid reaction. Eur J Intern Med 2006;17: 394-8

5 De Toledo FG, Symes SN. Leukemoid reaction due to Clostridium dificile infection in acquired immunodeficiency syndrome: two case reports and a review of the literature. South Med J 2004;97:388-92

6 Au WY, Ma SK, Kwong YL. Disseminated hepatosplenic mycobacterial infection masking myeloproliferative diseases as leukemoid reaction: a diagnostic pitfall. Leuk Lymphoma 2001;42:805-8

7 Halkes CJ, Dijstelbloem HM, Eelkman Rooda SJ, Kramer MH. Extreme leucocytosis: not always leukaemia. Neth J Med 2007;65:248-51

8 Nasser SM, Choudry UH, Nielsen GP, Ott MJ. A leukemoid reaction in a patient with a dedifferentiated liposarcoma. Surgery 2001;129:765-7

9 Nimieri HS, Makoni SN, Madziwa FH, Nemiary DS. Leukemoid reaction response to chemotherapy and radiotherapy in a patient with cervical carcinoma. Ann Hematol 2003;82:316-7

10 Jazayeri S, Tatou E, Cheynel N, Becker F, Brenot R, David M. A spontaneous rupture of the external iliac vein revealed as a phlegmasia cerulea dolens with acute lower limb ischemia: case report and review of the literature. J Vasc Surg 2002;35:999-1002

11 Marinella MA. Extreme leukemoid reaction associated with retroperitoneal hemorrhage. Arch Intern Med 1998; 158:300-1

12 Morales AM, Hashimoto LA, Mokhtee D. Alcoholic hepatitis with leukemoid reaction after surgery. J Gastrointest Surg 2006;10:83-5

13 Sakka V, Tsiodras S, Giamarellos-Bourboulis J, Giamarellou $H$. An update on the etiology and diagnostic 
evaluation of a leukemoid reaction. Eur J Intern Med 2006;17:394-8

14 Nomoto S, Kitagawa T, Ikebukuro E, Ito M, Taguchi T. Thrombosis of portal vein, splenic artery and vein with leukemoid reaction as a complication of gastric ulceration. A case report (author's translation). Nippon Shokakibyo Gakkai Zasshi 1977;74:790-4

15 Berk DR, Ahmed A. Portal, splenic, and superior mesenteric vein thrombosis in a patient with latent essential thrombocythemia and hyperhomocysteinemia. J Clin Gastroenterol 2006;40:227-8

16 Buzas C, Sparchez Z, Cucuianu A, Manole S, Lupescu I, Acalovschi M. Budd-Chiari syndrome secondary to polycythemia vera. A case report. J Gastrointestin Liver Dis 2009;18:363-6

17 Goldhaber SZ, Piazza G. Optimal duration of anticoagulation after venous thromboembolism. Circulation 2011;123:664-7 